\title{
Brussels sprouts, inulin and fermented milk alter the faecal microbiota of human microbiota-associated rats as shown by PCR-temporal temperature gradient gel electrophoresis using universal, Lactobacillus and Bifidobacterium 16S rRNA gene primers
}

\author{
Christèle Humblot, Aurélia Bruneau, Malène Sutren, Evelyne F. Lhoste, Joël Doré, Claude Andrieux \\ and Sylvie Rabot* \\ National Institute for Agronomic Research (INRA), Unit on Ecology and Physiology of the Digestive Tract (UEPSD), \\ 78352 Jouy-en-Josas Cedex, France \\ (Received 16 May 2004 - Revised 9 November 2004 - Accepted 11 November 2004)
}

\begin{abstract}
We investigated the effect of Brussels sprouts, inulin and a fermented milk on the faecal microbiota diversity of human microbiota-associated (HMA) rats by PCR-temporal temperature gradient gel electrophoresis (PCR-TTGE) using universal and group-specific 16S rRNA gene primers. The HMA rats were submitted to a control diet for $10 \mathrm{~d}$ (initial time), then switched to the experimental diets for 4 weeks (final time). Using universal primers, the mean degree of similarity between all faecal samples at initial time was $80.8 \%$. In the group consuming the control diet throughout the experiment, the mean degree of similarity between the PCR-TTGE profiles at initial $v$. final time was $76.8 \%$, reflecting a spontaneous temporal variation. The mean degree of similarity between control and experimental groups at final time was lower, $72.4 \%, 74.4 \%$ and $75.6 \%$ for inulin, Brussels sprouts and fermented milk, respectively, indicating a dietary effect on the predominant populations. Using specific primers, bifidobacteria could be detected only in those rats that had consumed inulin, showing a specific increasing effect of this dietary compound. The Lactobacillus population was very heterogeneous at initial time but tended to homogenize within each dietary group. At final time, caecal contents were collected for analysis of SCFA and $\beta$-glucuronidase activity. Inulin and Brussels sprouts increased the butyrate and acetate proportion, respectively, while the fermented milk did not modify the caecal biochemistry. This experiment shows for the first time that cruciferous vegetables are able to alter the diversity and the metabolic activities of the digestive microbiota in HMA rats.
\end{abstract}

Human intestinal microbiota: Prebiotic: Probiotic: Brassica vegetables: TTGE

Epidemiological studies implicate heterocyclic amines (HA), mutagenic/carcinogenic compounds commonly found in fried meats and fish, as risk factors in the aetiology of human colon cancer (Felton et al. 2002). For several years, strong efforts have been made to identify dietary constituents that could protect against the genotoxic and carcinogenic effects of HA. Among them, oligosaccharides, fermented milks containing lactic acid bacteria and Brassica vegetables have shown chemoprotection in laboratory rodents (Reddy \& Rivenson, 1993; Schwab et al. 2000; Kassie et al. 2002, 2003; Tavan et al. 2002; Humblot et al. 2004) and different mechanisms have been proposed to explain their protective effect. Chemoprotection by Brassica vegetables such as Brussels sprouts, red cabbage and garden cress is often paralleled with a significant increase of hepatic UDP-glucuronosyl transferase (EC 2.4.7.17) activity (Kassie et al. 2002, 2003). Since conjugation with glucuronic acid is the main detoxification pathway of HA, this increase could account for the protective effect of Brassica vegetables. On the other hand, it has been hypothesized that hydrolysis of glucuronidated HA by bacterial $\beta$-glucuronidase (EC 3.2.1.31) in the colonic lumen leads to the release of reactive metabolites that can cause DNA damage in the colonic mucosa or in the liver, which they can reach via the enterohepatic circulation. Therefore, the decreasing effect of fructose polymers such as inulin on the $\beta$-glucuronidase activity of intestinal microbiota may contribute to their protective effect against the genotoxicity of HA (Rowland et al. 1998; Humblot et al. 2004). With regard to fermented milks, their protective action cannot be related to any modification of mammalian or bacterial enzymes involved in the activation and detoxification of HA (Humblot et al. 2004); hence, direct binding of these compounds by lactic acid bacteria, which has been described in several in vitro studies, is still put forward as the most likely explanation (Knasmüller et al. 2001).

Nevertheless, data on the impact of the intestinal microbiota on the genotoxicity of HA in vivo are still scarce. Kassie et al. (2001) have shown that the extent of HA-induced DNA damage in colonocytes and hepatocytes, measured using the comet assay, was 2-3-fold higher in human microbiota-associated rats (HMA

\footnotetext{
Abbreviations: DGGE, denaturing gradient gel electrophoresis; HA, heterocyclic amine; HMA rat, human microbiota-associated rat; IQ, 2-amino-3-methylimidazo[4,5$f$ ]quinoline; MRS, De Man, Rogosa and Sharpe; TGGE, temperature gradient gel electrophoresis; TTGE, temporal temperature gradient gel electrophoresis. * Corresponding author: Dr Sylvie Rabot, fax +33 1346524 62, email Sylvie.Rabot@jouy.inra.fr
} 
rats), and 4-5-fold higher in conventional rats, than in germ-free companions. These findings highlight the crucial impact of intestinal microbiota on the genotoxicity of HA and suggest the hypothesis that fermented milks, but also other dietary constituents, could be protective through an alteration of the intestinal microbial ecology. In a recent study (Humblot et al. 2004), we have shown that Brussels sprouts, inulin and a fermented milk were capable of reducing up to $70 \%$ the extent of DNA damage induced by 2-amino-3-methylimidazo[4,5- $f$ ]quinoline (IQ) in the colonic mucosa and in the liver of HMA rats. In this context, the aim of the present study was to investigate the influence of these dietary constituents on the phylogenetic diversity of the faecal microbiota of HMA rats.

Our present knowledge of the intestinal microbiota is largely based on cultivation studies but, according to estimates, up to $85 \%$ of the entire microbial population in the human intestine might be uncultured (Suau et al. 1999). In order to overcome the limitations associated with culturing techniques, molecular biological methods are increasingly being applied to study the intestinal microbial ecology (Vaughan et al. 2000). One of the most widely used approaches in ecological studies has been the use of 16S rRNA and its encoding genes as target molecules (Amann et al. 1995). Specific PCR primers and probes can be designed based on the variable regions of this molecule to detect predominant bacterial populations as well as specific genera (Zoetendal et al. 1998; Satokari et al. 2001; Walter et al. 2001). The amplified fragments of $16 \mathrm{~S}$ rRNA are then separated in a sequence-specific manner in temperature gradient gel electrophoresis (TGGE) or denaturing gradient gel electrophoresis (DGGE) (Muyzer et al. 1993; Muyzer \& Smalla, 1998). These techniques have already been successfully applied to monitor bacterial populations in human faecal samples (Zoetendal et al. 1998; Satokari et al. 2001; Walter et al. 2001) and in the faeces of gnotobiotic rats harbouring human intestinal microbiota (Gérard et al. 2004).

In the experiment reported here, we applied PCR-temporal temperature gradient gel electrophoresis (TTGE) analysis to monitor the predominant population and specific subpopulations of the faecal microbiota of HMA rats, before and after consumption of Brussels sprouts, fermented milk and inulin. For this purpose, we used a combination of universal and group-specific (targeting the genus Bifidobacterium and the Lactobacillus group) $16 \mathrm{~S}$ rRNA gene primers. In parallel, intestinal microbiota-associated metabolic biomarkers of colon cancer, namely $\beta$-glucuronidase and SCFA, were measured.

\section{Material and methods}

\section{Chemicals}

Polypeptone and papaic digest of soyabean meal were purchased from AES Laboratoire (Combourg, France), yeast extract and De Man, Rogosa and Sharpe (MRS) culture medium from Difco (Le Pont de Claix, France), and sodium glycerophosphate and ascorbic acid from VWR (Fontenay-sous-Bois, France). The agarose and ethidium bromide came from Euromedex (Mundolsheim, France), the Tris(hydroxymethyl)aminomethane-borate-ethylenediaminetetraacetic acid from Gibco (Invitrogen, Cergy-Pontoise, France), the acrylamide from Eurobio (Les Ulis, France), the extract of meat dry and the urea from Merck (Paris, France). Tris(hydroxymethyl)aminomethane-acetate-ethylenediaminetetraacetic acid was purchased from Bio-Rad (Marnes-la-Coquette, France) and SYBR
Green I Nucleic Acid Gel Stain was from Roche Diagnostics (Mannheim, Germany). All other chemicals came from Sigma-Aldrich (Saint-Quentin-Fallavier, France).

\section{Animals}

Forty-eight germ-free male Fischer 344 rats, aged 3 months at the start of the experiment, were provided by the breeding facilities of the Unit on Ecology and Physiology of the Digestive Tract of INRA (Jouy-en-Josas, France). Throughout the study, animals were housed three per cage in flexible-film isolators (La Calhène, Vélizy, France) and were given free access to their diet and to sterilized tap water. The room harbouring the isolators was maintained at constant temperature and humidity $\left(21 \pm 1^{\circ} \mathrm{C}, 50 \pm 5 \%\right)$ with a $12 \mathrm{~h}$ light/dark cycle.

\section{Diets}

The diets were semi-synthetic designed to simulate a human-type diet. Therefore, lipids and proteins of both animal and vegetable origins, as well as saccharose and cooked starch, were included. The control diet consisted of $28.985 \%$ (w/w) maize starch, $29 \%$ mashed potato, $5 \%$ saccharose, $5 \%$ casein, $12 \%$ soya isolate, $3 \%$ maize oil, $3 \%$ lard, $0.015 \%$ cholesterol, $6 \%$ cellulose and $8 \%$ mineral and vitamin additives (Lhoste et al. 2003). Analytical compounds of DM were crude proteins $18 \%$, crude fat $8 \%$, ash $6 \%$, carbohydrates $68 \%$ (energy: $19.33 \mathrm{MJ} / \mathrm{kg} \mathrm{DM}$; Eurofins Scientific Analytics, Nantes, France). In the inulin diet, saccharose and $5 \%$ maize starch were replaced by $10 \%$ Raftilo$\mathrm{se}^{\circledR}$ Synergy 1 (Orafti, Tienen, Belgium): $100 \mathrm{~g}$ Raftilose ${ }^{\circledR}$ Synergy 1 provided $97 \mathrm{~g}$ carbohydrates (energy $693 \mathrm{~kJ}$ ), among which $89 \mathrm{~g}$ were inulin; protein and fat were absent and vitamin and mineral content was negligible. In the Brussels sprouts diet, $4 \%$ maize starch, $4 \%$ mashed potato and $2 \%$ soya isolate were replaced by $10 \%$ freeze-dried Brussels sprouts (Brassica oleracea variety Cyrus; Novartis Seeds, Enkhuizen, the Netherlands) containing $24 \mu \mathrm{mol}$ glucosinolates/g DM (Rouzaud et al. 2003). Brussels sprouts are usually cooked before consumption; this process denaturates plant myrosinase (EC 3.2.1.147) so that glucosinolates reach the colon, where they are hydrolysed by the intestinal microbiota into bioactive isothiocyanates (Johnson, 2002). Therefore, Brussels sprouts were treated to denaturate myrosinase before inclusion in the diet as described by Krul et al. (2002) and Rouzaud et al. (2003). The fermented milk diet was a control diet supplemented with the fermented milk Actimel ${ }^{\circledR}$ purchased from a local market $(1 \mathrm{ml}$ fermented milk for $1 \mathrm{~g}$ dietary powder). Actimel ${ }^{\circledR}$ contains three species of lactic acid bacteria, namely Lactobacillus bulgaricus, Lactobacillus casei and Streptococcus thermophilus. Five batches of Actimel ${ }^{\circledR}$ were used throughout the experiment; each batch was numbered for L. bulgaricus on acid MRS ( $55 \mathrm{~g} / \mathrm{l}, \mathrm{pH} 5.4$ ), for L. casei on MRS (55 g/l, $\mathrm{pH} 6.5)$ and for $S$. thermophilus on M17 saccharose (polypeptone $5 \mathrm{~g} / \mathrm{l}$, papaic digest of soyabean meal $5 \mathrm{~g} / 1$, extract of meat dry $4 \mathrm{~g} / 1$, yeast extract $2.5 \mathrm{~g} / 1$, saccharose $5 \mathrm{~g} / \mathrm{l}$, sodium glycerophosphate $19 \mathrm{~g} / \mathrm{l}$, ascorbic acid $0.5 \mathrm{~g} / \mathrm{l}$ and magnesium sulphate $0.25 \mathrm{~g} / \mathrm{l}$ ). The results are 2.2 (SEM $1.0) \times 10^{8}, 5.0(\operatorname{SEM~} 0.2) \times 10^{7}$ and $9.0(\operatorname{SEM~} 0.4) \times 10^{7}$, respectively for L. bulgaricus, L. casei and S. thermophilus. Actimel ${ }^{\circledR}$ typically contains (per $100 \mathrm{ml}$ ): $2.8 \mathrm{~g}$ proteins, $1.6 \mathrm{~g}$ fat and 
$14.3 \mathrm{~g}$ carbohydrates (energy $350 \mathrm{~kJ}$ ); saccharose, lactose and glucose accounted for 68,21 and $2 \%$ of carbohydrates, respectively.

All dietary powders were manufactured by U.A.R. (Villemoisson-sur-Orge, France), packed in double-vacuum bags and sterilized by $\gamma$-irradiation at $45 \mathrm{kGy}$ (U.A.R.). Diets were prepared freshly every $2 \mathrm{~d}$ in the isolators by mixing thoroughly $50 \mathrm{~g}$ dietary powder with $100 \mathrm{ml}$ water (control, inulin and Brussels sprouts diets) or with $50 \mathrm{ml}$ fermented milk and $50 \mathrm{ml}$ water (fermented milk diet).

\section{Experimental design}

All procedures were carried out in accordance with the European guidelines for the care and use of laboratory animals.

The germ-free rats were randomly allocated to four dietary groups (control, Brussels sprouts, fermented milk and inulin groups) housed in four distinct isolators (twelve animals per group, three animals per cage). On day 1, all rats were inoculated orally with the whole faecal microbiota of a healthy adult man as described previously (Roland et al. 1996) and offered the control diet. On day 10, one faecal pellet was freshly collected from each HMA rat; pellets were pooled by cage and stored at $-80^{\circ} \mathrm{C}$ until analysis of the microbiota by PCR-TTGE (initial time). HMA rats of the Brussels sprouts, fermented milk and inulin groups were thereafter switched to the experimental diets. Four weeks later, faecal pellets were again collected, pooled by cage and stored at $-80^{\circ} \mathrm{C}$ until PCR-TTGE analysis (final time). HMA rats were then killed by $\mathrm{CO}_{2}$ inhalation, the caecal $\mathrm{pH}$ was measured, and caecal contents were weighed and distributed into several vials stored at $-80^{\circ} \mathrm{C}$ for SCFA and $\beta$-glucuronidase analyses.

\section{DNA extraction, PCR and temporal temperature gradient gel} electrophoresis analysis of the faecal samples and the fermented milk

Total DNA was extracted from the faecal samples and from the fermented milk using an amalgamator with glass beads (Minibeadbeater-8, Biospec products, VWR) as described by Godon et al. (1997). The V6 to V8 regions of the 16S rRNA genes were amplified by PCR using the HotStar Taq Master Mix Kit (Qiagen, Courtabœuf, France) in a PCT 100 thermocycler (MJ Research, USA) (Gérard et al. 2004). The universal primers U968-GC and L1401 originally described by Zoetendal et al. (1998) were used to amplify non-selectively the bacteria; the primers Lac 352 and Lac 679 initially designed by Walter et al. (2001) were used to amplify selectively the lactobacilli; in order to examine the bifidobacteria we used the primers Bif 164 and Bif 662 initially designed by Satokari et al. (2001). TTGE analysis of the amplicons was performed using the Dcode ${ }^{\mathrm{TM}}$ Universal Mutation Detection System (Bio-Rad, Paris, France) as described by Seksik et al. (2003). Electrophoresis was run at a fixed voltage of $76 \mathrm{~V}$ for $16 \mathrm{~h}$ with an initial temperature of $66^{\circ} \mathrm{C}$ for universal and Bifidobacterium amplicons and of $64^{\circ} \mathrm{C}$ for Lactobacillus amplicons, and a ramp rate of $0 \cdot 2^{\circ} \mathrm{C} / \mathrm{h}$. For better resolution, the voltage was fixed at $20 \mathrm{~V}$ for $15 \mathrm{~min}$ at the beginning of electrophoresis (Zoetendal et al. 1998; Satokari et al. 2001; Walter et al. 2001; Seksik et al. 2003). After the completion of the electrophoresis, gels were stained in the dark by immersion in a solution of SYBR Green I Nucleic Acid Gel Stain and read on a Storm system (Molecular Dynamics).
Analysis of SCFA and $\beta$-glucuronidase activity in the caecal contents

All analyses were performed in duplicate. $\beta$-Glucuronidase activity was measured spectrophotometrically $(\lambda=400 \mathrm{~nm})$ by the rate of release of $p$-nitrophenol from the $p$-nitrophenylglucuronide as described by Andrieux et al. (1998). Enzymatic activity was expressed as $\mu \mathrm{mol}$ of product formed per min and per $\mathrm{g}$ of wet caecal content. SCFA were analysed after water extraction of acidified samples, using a gas-liquid chromatograph (Nelson 1020, Perkin Elmer, Saint-Quentin-en-Yvelines, France) equipped with a flame-ionization detector and a wide-bore column $(15 \mathrm{~m} \times 0.53 \mathrm{~mm})($ FSCAP Nukol, Supelco, Saint-Quentin-Fallavier, France) impregnated with SP 1000. Carrier gas (He) flow rate was $10 \mathrm{ml} / \mathrm{min}$, inlet temperature $100^{\circ} \mathrm{C}$ and detector temperature $280^{\circ} \mathrm{C}$. 2-Ethylbutyrate was used as the internal standard (Rabot et al. 2000).

\section{Calculations and statistical analyses}

Inter-individual variability and the effects of time and diet on the faecal microbiota diversity were investigated. To determine the inter-individual variability, TTGE profiles of all faecal samples ( $n$ 16) were compared at initial time; the effect of time was consequently assessed by comparing the TTGE profiles of control samples $(n 4)$ at initial $v$. final time; eventually, the dietary effect was examined by comparing at final time the control TTGE profiles $(n 4)$ with those of rats consuming the experimental diets. Comparisons of TTGE profiles were performed using the GelCompar $I^{\mathrm{TM}}$ software (version 2.0, Applied Maths, Kortrijk, Belgium). Analysis included calculation of the number, position and intensity of bands for each lane, and between-pattern comparisons using the Pearson correlation coefficient calculated as a measure of the degree of similarity; a value of 100 indicates that the samples are identical.

The effect of diet on caecal SCFA and $\beta$-glucuronidase activity was analysed using a one-way ANOVA. When ANOVA indicated significant differences, groups receiving the inulin, Brussels sprouts and fermented milk diets were each compared with the group fed on the control diet using the Dunnett's test. Statistical significance was set at $P<0 \cdot 05$. Calculations were performed using the Statview ${ }^{\circledR}$ software (version 5.0, SAS Institute, Cary, NC, USA). All data were expressed as mean values and standard errors of the means.

\section{Results}

Effect of the diets on the PCR-temporal temperature gradient gel electrophoresis profiles of human microbiota-associated rats' faecal microbiota using universal primers

PCR-TTGE analysis of the faecal microbiota using universal primers results in complex profiles that represent the predominant microbial communities, and in which each band represents at least a single bacterial 16S rDNA. Fig. 1 shows an example of the TTGE profiles obtained at the initial time of the experiment, i.e. $10 \mathrm{~d}$ after all initially germ-free rats had been inoculated with the human faecal microbiota and fed on the control diet. At this time, the mean Pearson correlation coefficient between all samples was 80.8 (SEM 1.0) \%; this figure represents the interindividual variability at initial time. To monitor the spontaneous evolution of the predominant species-pattern over time, we 


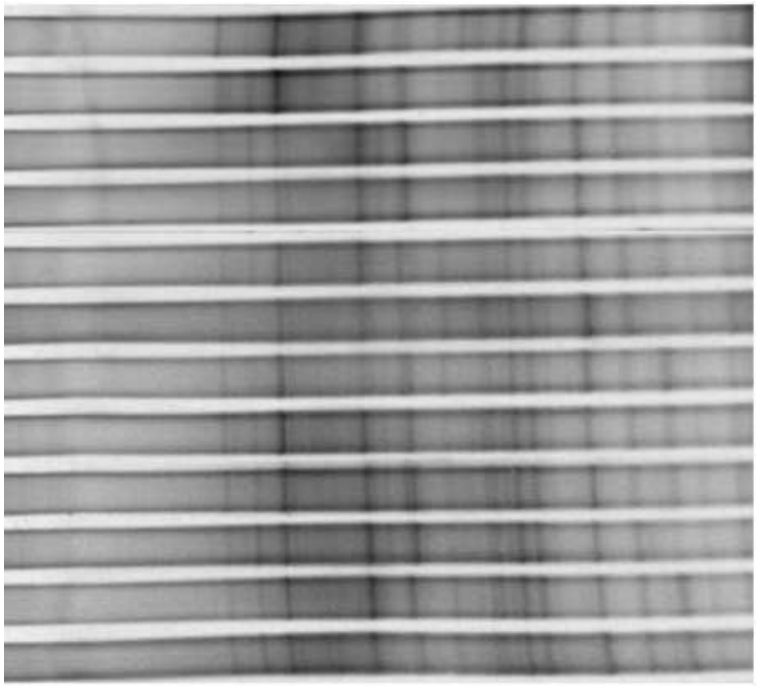

Fig. 1. Example of faecal PCR-temperature gradient gel electrophoresis profiles obtained with universal primers in human microbiota-associated rats. Samples were collected $10 \mathrm{~d}$ after initially germ-free rats had been inoculated with the human faecal microbiota and fed continuously on the control diet (initial time). For details of diets and procedures, see p. 678.

compared the TTGE profiles of the control group at initial time with those of the same group at final time, i.e. after 4 weeks consumption of the control diet. The mean Pearson correlation coefficient was 76.8 (SEM 2.5)\% (Fig. 2). At final time, the mean Pearson correlation coefficients between the control group and each of the experimental groups were 72.4 (SEM 1.0)\%, 74.4 (SEM 1.0) \% and 75.6 (SEM 2.0) \%, respectively, for inulin, Brussels sprouts and fermented milk (Fig. 2). These data indicate that the faecal microbiota diversity was more altered after consumption of the experimental diets than after continuous feeding on the control diet. In conclusion, consumption of experimental diets for 4 weeks modified the pattern of the predominant populations in the faeces of the HMA rats.
Effect of the diets on the PCR-temporal temperature gradient gel electrophoresis profiles of human microbiota-associated rats' faecal microbiota using Bifidobacterium specific primers

No PCR amplicon of Bifidobacterium 16S rDNA could be obtained at the initial time, regardless of dietary groups (data not shown), and at the final time in the groups consuming the control, Brussels sprouts and fermented milk diets (Fig. 3). On the contrary, faecal samples collected at the final time from rats receiving the inulin-diet contained Bifidobacterium species at a predominant level, as shown by the PCR products obtained with Bifidobacterium specific primers (Fig. 3). TTGE separation of these PCR products led to a similar pattern in all samples with one band, representing at least one species of Bifidobacterium, being particularly stimulated by the inulin consumption (Fig. 4).

Effect of the diets on the PCR-temporal temperature gradient gel electrophoresis profiles of human microbiota-associated rats' faecal microbiota using Lactobacillus specific primers

Fig. 5 shows for each dietary group the TTGE profiles obtained with Lactobacillus specific primers at initial $v$. final time. At the start of the experiment, all faecal samples exhibited various one to three band-patterns, apart from one sample of the control group that presented no band at all. Four weeks later, all control samples shared a common two to three band-pattern that differed from those observed at the initial time. In the inulin group, a new band, common to all samples, appeared after 4 weeks of consumption of the experimental diet, while the initial bands had almost totally disappeared. Similarly, an extensive reorganization of the Lactobacillus population occurred in the Brussels sprouts group as the initial one to three band-pattern totally disappeared, while two new bands appeared. In the fermented milk group as well, a new band appeared in all samples whereas several initial bands were not visible anymore.

The fermented milk profile is composed of two bands corresponding to the species L. bulgaricus and L. casei. A band

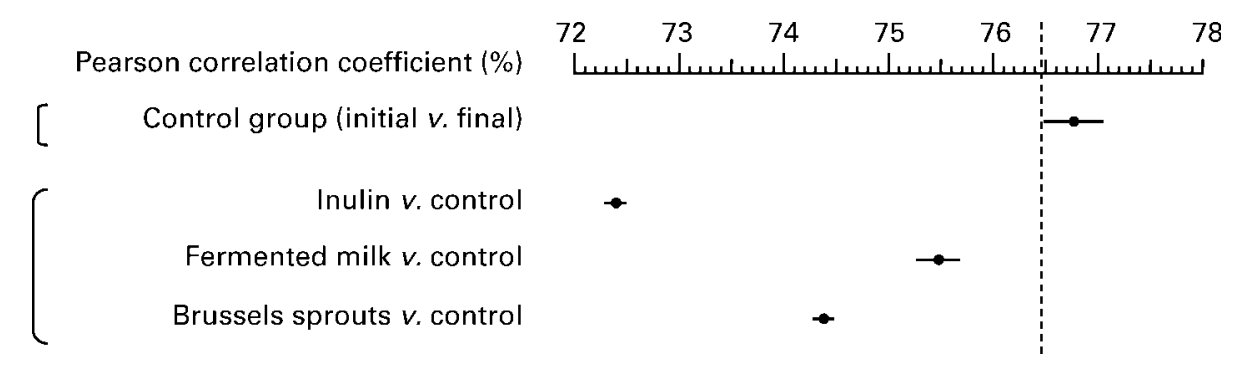

Fig. 2. Pearson correlation coefficients between PCR-temperature gradient gel electrophoresis profiles obtained with universal primers (means (bullets) with their standard errors). At initial time, the mean Pearson correlation coefficient between all samples was 80.8 (SEM 1.0)\% (inter-individual variability). The control group was compared at initial $v$. final time to monitor the spontaneous evolution of the pattern with time (effect of time). Groups receiving the inulin, fermented milk and Brussels sprouts diets were compared with the control group at the final time (effect of the experimental diets). For details of diets and procedures, see p. 678.

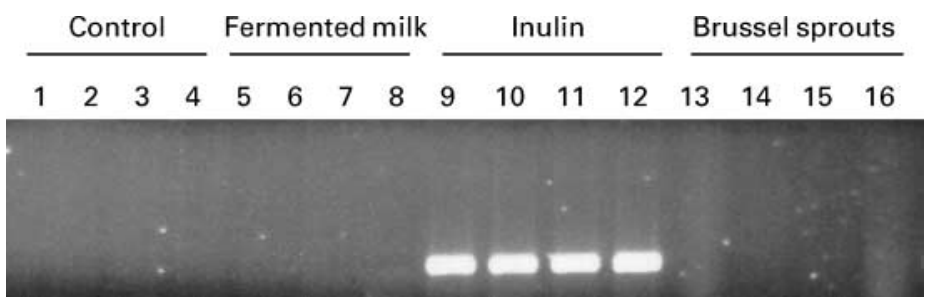

Fig. 3. PCR products of amplification using Bifidobacterium specific primers. Lanes $1-4,5-8,9-12$ and $13-16$ are PCR products from human microbiota-associated rats fed on the control, the fermented milk, the inulin and the Brussels sprouts diet, respectively (final time). For details of diets and procedures, see p. 678. 


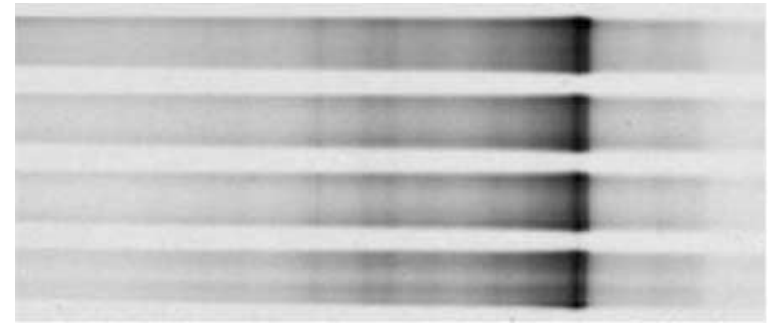

Fig. 4. PCR-temperature gradient gel electrophoresis profiles obtained with Bifidobacterium primers in human microbiota-associated rats fed on the inulindiet for 4 weeks (final time). For details of diets and procedures, see p. 678 .

co-migrating with $L$. bulgaricus was present at initial time in several faecal samples; at final time, this band persisted only in the faeces of HMA rats fed on the fermented milk diet. Interestingly, no band co-migrating with $L$. casei could ever be seen, whatever the sample and the time of the experiment.

On the whole, the Lactobacillus population was remarkably heterogeneous at the beginning of the experiment and a trend towards homogenization was observed with time, with specific features within each dietary group.
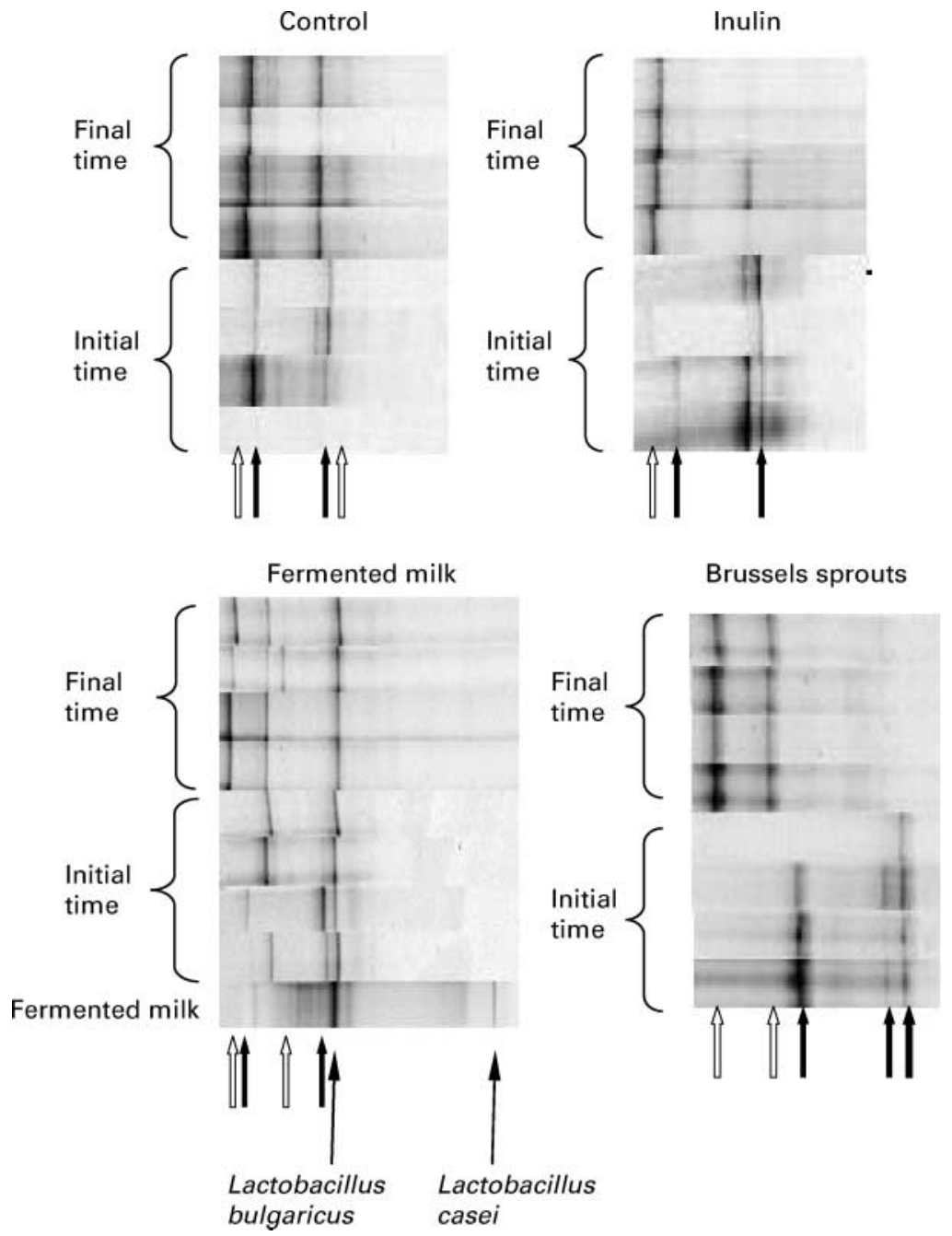

Effect of the diets on caecal weight, $\mathrm{pH}, \beta$-glucuronidase activity and fermentative metabolites

None of the diets modified the caecal pH (Table 1) and the caecal weight increased only after inulin consumption (136\% of the control value; $P<0 \cdot 05$; Table 1$)$. The $\beta$-glucuronidase activity and the total SCFA concentration were similar regardless of the diet while the SCFA profile differed significantly in the groups fed on the inulin and the Brussels sprouts diets compared with the control group. Inulin increased the proportion of butyrate (179\% of the control value; $P<0.05$; Table 1 ) at the expense of acetate and branched-chain fatty acids (respectively $89 \%$ and $44 \%$ of the control values; $P<0.05$; Table 1 ). Brussels sprouts increased the proportion of acetate $(108 \%$ of the control value; $P<0.05$; Table 1) at the expense of branched-chain fatty acids (41\% of the control value; $P<0 \cdot 05$; Table 1 ).

\section{Discussion}

HMA rats are now a well-validated model for experimental studies aimed at evaluating the effects of functional foods in gastrointestinal physiology or in carcinogenesis (Djouzi et al. 1997; Kleessen

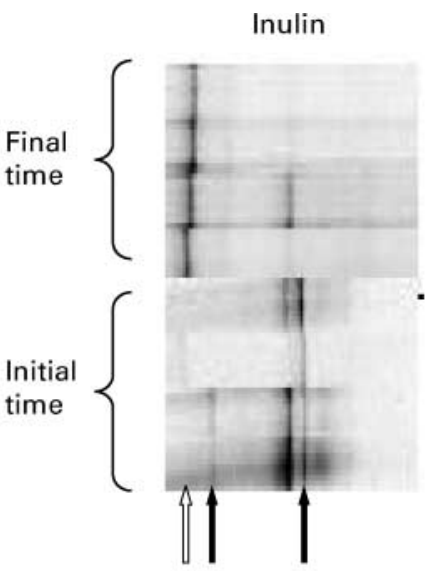

Brussels sprouts

Fig. 5. PCR-temperature gradient gel electrophoresis profiles obtained with Lactobacillus primers from the fermented milk and from human microbiota-associated rats at initial time (consumption of the control diet for $10 \mathrm{~d}$ ) and at final time (consumption of the experimental diets for 4 weeks). the bands present at the initial time but absent at the final time; $\hat{\imath}$ the new bands detected at the final time. For details of diets and procedures, see p. 678 
Table 1. Effect of the Brussels sprouts, fermented milk and inulin diets on the weight and $\mathrm{pH}$ of the caecal content, and on the caecal $\beta$-glucuronidase activity and SCFA concentration and profile in human microbiota-associated rats $\dagger$

(Mean values with their standard errors of the means)

\begin{tabular}{|c|c|c|c|c|c|c|c|c|}
\hline & \multicolumn{2}{|c|}{ Control diet } & \multicolumn{2}{|c|}{ Brussels sprouts diet } & \multicolumn{2}{|c|}{ Fermented milk diet } & \multicolumn{2}{|c|}{ Inulin diet } \\
\hline & Mean & SEM & Mean & SEM & Mean & SEM & Mean & SEM \\
\hline Caecal pH & $6 \cdot 77$ & 0.14 & $6 \cdot 64$ & $0 \cdot 10$ & 6.58 & $0 \cdot 16$ & $6 \cdot 51$ & 0.11 \\
\hline$\beta$-Glucuronidase $(\mu \mathrm{mol} / \mathrm{min} / \mathrm{g}) \ddagger$ & 0.31 & 0.04 & 0.28 & 0.04 & 0.27 & 0.04 & 0.34 & 0.08 \\
\hline $\operatorname{SCFA}(\mu \mathrm{mol} / \mathrm{g}) \ddagger$ & $70 \cdot 17$ & $10 \cdot 87$ & 87.51 & 4.50 & $66 \cdot 28$ & 7.44 & $87 \cdot 03$ & $7 \cdot 19$ \\
\hline \multicolumn{9}{|l|}{ SCFA profile $(\mu \mathrm{mol} / 100 \mu \mathrm{mol})$} \\
\hline Propionate & 15 & 1 & 13 & 1 & 15 & 2 & 11 & 1 \\
\hline Butyrate & 15 & 1 & 13 & 1 & 15 & 2 & $27^{*}$ & 1 \\
\hline Valerate+caproate & 1 & 0 & 1 & 0 & 1 & 0 & 1 & 0 \\
\hline BCFA & 2 & 0 & $1^{*}$ & 0 & 2 & 0 & $1^{*}$ & 0 \\
\hline
\end{tabular}

BCFA, branched-chain fatty acids.

${ }^{*}$ Mean values were significantly different from the control group (Dunnett's test, ${ }^{*} P<0.05$ ).

$\dagger$ For details of diets and procedures, see Material and methods.

$\ddagger$ Results are expressed as g caecal wet weight. All analyses were made in duplicate.

et al. 2001; Humblot et al. 2004; Imaoka et al. 2004). In particular, this model facilitates the investigations on the interactions between food compounds and the human gut microbiota, since it is easier to control and alter animal diets than those of human subjects. Furthermore, it enables there to be several repetitions of the same microbiota for one experimental diet, thus avoiding the fluctuations observed by several authors in human experiments (Tannock, 2001; Zoetendal et al. 2001). A major proportion of the gut microbiota is currently uncultivable but the use of molecular techniques allows this difficulty to be overcome (Tannock, 2001). In this respect, this study is, to our knowledge, the first one that exploits PCR-TTGE analysis to examine the effect of functional foods on the faecal microbial population of HMA rats, using universal and group-specific primers.

Among the three foodstuffs that were studied here, i.e. inulin, Brussels sprouts and a fermented milk, inulin was undoubtedly the most effective with regard to modifications of the predominant faecal bacterial populations. The mean degree of similarity between inulin and control samples was $72.4 \%$, a value noticeably distant from $76.8 \%$, i.e. the mean coefficient between control samples at initial $v$. final time that reflects the spontaneous evolution of the predominant microbiota-profile with time. The marked bifidogenic effect of inulin probably accounts, at least partly, for this reshaping of the overall PCR-TTGE profile. Fructose polymers with short chains (oligofructose) or long chains (inulin) have been extensively investigated for their selective stimulation of Bifidobacterium (Roberfroid, 2001; Cummings \& MacFarlane, 2002). Campbell et al. (1997) showed by enumeration on selective media that oligofructose increases the Bifidobacterium in the caecum of conventional rats; more recently, Kleessen et al. (2001) have used fluorescent in situ hybridization to demonstrate that inulin and oligofructose are both able to increase the Bifidobacterium population in the digestive tract of HMA rats. Using PCR-TTGE, we confirm this increase and we show that several bands accounting for different Bifidobacterium species become predominant following 4 weeks of inulin consumption, one of them being particularly stimulated. Bifidobacteria have been studied by several authors for their ability to detoxify HA (Knasmüller et al. 2001). Most of the studies take place in vitro, and the most important mechanism appears to be direct binding of HA to the wall of the bacterial cells (Zhang \& Ohta, 1993;
Orrhage et al. 1994; Lankaputhra \& Shah, 1998). Recently, Tavan et al. (2002) have reduced by $96 \%$ the frequency of HAinduced colonic aberrant crypt foci by feeding rats with a fermented milk containing the species Bifidobacterium animalis (Tavan et al. 2002); similarly, Zsivkovits et al. (2003) have obtained a reduction of HA-induced DNA damage in the colon of rats after gavage with Bifidobacterium longum (Zsivkovits et al. 2003). Because of the heterogeneousness of the Lactobacillus group at the start of the experiment and its temporal variation in the control group, it is difficult to conclude whether the shifts observed in the inulin group arise from a specific dietary effect or from a spontaneous temporal instability. These observations are consistent with those of Vanhoutte et al. (2004), who have lately investigated the temporal stability of different populations of the human faecal microbiota using DGGE; they concluded that the Lactobacillus group tends to show strong temporal variations whereas other autochthonous groups, e.g. the genus Bifidobacterium, do not undergo major population shifts in time. We confirm otherwise the butyrogenic effect of inulin (Andrieux et al. 1991; Kleessen et al. 2001). Since butyrate has been consistently found to cause apoptosis of cancerous cells (Avivi-Green et al. 2002), this inulin property supports the current insights on its chemopreventive potential against the development of colon cancer. We did not find any modification of the $\beta$-glucuronidase activity. Actually it must be emphasized that the modulating effect of inulin on this enzyme activity, which is considered to be a luminal biomarker of colon cancer, is not consistently reported throughout the literature (Djouzi \& Andrieux, 1997; Rowland et al. 1998; Hughes \& Rowland, 2001; Humblot et al. 2004).

Similar to inulin, Brussels sprouts modified the predominant populations of the faecal microbiota of HMA rats. Indeed, the mean degree of similarity between the PCR-TTGE profiles of control rats and those of companions fed for 4 weeks on the Brussels sprouts diet was $74.4 \%$, a figure below the $76.8 \%$ threshold value. No increasing effect could be observed on the Bifidobacterium population and, once again, because of a temporal variation background, the shifts of the Lactobacillus group could not be confidently ascribed to a dietary effect. To our knowledge, this study is the first evidence of the effect of a cruciferous vegetable on the composition of faecal microbiota of human origin. Besides these modifications, the consumption of Brussels sprouts led to an 
alteration of the SCFA pattern, increasing acetate at the expense of branched-chain fatty acids, but did not change the $\beta$-glucuronidase activity. In a previous study, we had shown that Brussels sprouts reduce the DNA damage induced by IQ in the colon of HMA rats (Humblot et al. 2004). The chemoprotection of cruciferous vegetables against the genotoxicity of HA is usually attributed to an induction of the UDP-glucuronosyl transferase, a key enzyme of the detoxification pathway of HA (Kassie et al. $2002,2003)$. The present study gives a new insight into the biological activities of cruciferous vegetables. However, whether these modifications of the predominant bacterial community and its fermentation pattern play a role in the chemopreventive effect of these foodstuffs remains to be established.

Unlike inulin and Brussels sprouts, the fermented milk Actimel ${ }^{\circledR}$ did not induce a noticeable modification of the predominant bacterial population pattern (mean degree of similarity between the control and treated groups: $75.6 \%$ ). Again, the heterogeneousness of the Lactobacillus population at initial time and the temporal variation observed in the control group prevent any firm conclusion on a possible dietary effect. A band co-migrating with the Lactobacillus acidophilus present in the fermented milk was observed in the faeces of several HMA rats at the start of the experiment, when all animals consumed the control diet. This band was present in all the samples collected from HMA rats fed for 4 weeks on the fermented milk diet. Nevertheless, and because of its sporadic presence at initial time, it is impossible to assign this DNA band to the Lactobacillus acidophilus of the fermented milk. This difficulty has already been noticed by certain authors in the course of human studies, where the initial presence of a band co-migrating with the Lactobacillus strain of the fermented milk disturbs interpretation of the data (Kimura et al. 1997; Tannock et al. 2000). On the whole, in our experimental conditions, the consumption of the fermented milk Actimel ${ }^{\circledR}$ resulted in a slight modification of the predominant faecal microbiota, without any marked influence on Bifidobacterium and Lactobacillus populations. In the same way, no consistent alterations of the caecal biochemistry could be observed following consumption of the fermented milk. This result contrasts with other studies in which consumption of the same fermented milk by HMA rats resulted in a decrease of the $\beta$-glucuronidase activity and a modification of the SCFA profile (Djouzi et al. 1997). However, consumption of this fermented milk by healthy infants remained ineffective on the SCFA profile and reduced the faecal $\beta$-glucuronidase activity mainly in those subjects whose basic activity was unusually high (Guérin-Danan et al. 1998). Therefore, it seems that the effect of functional foods on bacterial $\beta$-glucuronidase may depend on the basic activity of this enzyme in a given faecal microbiota.

The origin of the microbiota strongly influences the extent of the genotoxic effects of HA. For instance, DNA damage in the colonic mucosa following IQ treatment is twice greater in rats harbouring their natural microbiota than in HMA companions (Kassie et al. 2001). Focusing on the human colonic ecosystem, a recent study has shown that rats harbouring human microbiota from vegeterian subjects have $35 \%$ less IQ-induced DNA damage than rats inoculated with meat eaters' faecal microbiota (Kassie et al. 2004). In a previous study, we have demonstrated that diets containing inulin, Brussels sprouts or the fermented milk Actimel ${ }^{\circledR}$ protected HMA rats against the intestinal genotoxicity of IQ, with a $55 \%$ (Brussels sprouts) to $70 \%$ (inulin) reduction in the damage intensity. In the present study, inulin and, to a lesser extent, Brussels sprouts alter the profile of the predominant bacterial community of the human faecal ecosystem and, in the case of inulin, of the Bifidobacterium group. Therefore, it is possible that changes in the intestinal microbiota mediated by these foodstuffs contribute to their protective effects against HA genotoxicity.

\section{Acknowledgements}

The authors thank Rosa Durao for breeding the germ-free rats. Many thanks also to Dr Ruud Verkerk (University of Wageningen, the Netherlands) for the gift of the freeze-dried Brussels sprouts and to Dr Jan Van Loo (Orafti) for the Raftilose ${ }^{\circledR}$ Synergy 1. Dr Gérard Corthier, Dr Maria-José Flores-Sanabria and Dr Philippe Langella of INRA made helpful comments on the draft manuscript. This research was supported by the European Community under the RTD programme "Quality of Life and Management of Living Resources", QLK1-CT99-01 197, entitled "Heterocyclic Amines in Cooked Foods - Role in Human Health". Christèle Humblot acknowledges a PhD grant from the French Ministry of Education and Research.

\section{References}

Amann RI, Ludwig W \& Schleifer KH (1995) Phylogenetic identification and in situ detection of individual microbial cells without cultivation. Microbiol Rev 59, 143-169.

Andrieux C, Hibert A, Houari AM, Bensaada M, Popot F \& Szylit O (1998) Ulva lactuca is poorly fermented but alters bacterial metabolism in rats inoculated with human faecal flora from methane and nonmethane producers. J Sci Food Agric 77, 25-30.

Andrieux C, Lory S, Dufour-Lescoat C, de Baynast R \& Szylit O (1991) Physiological effects of inulin in germ-free rats and in heteroxenic rats inoculated with a human flora. Food Hydrocolloids 5, 49-56.

Avivi-Green C, Polak-Charcon S, Madar Z \& Schwartz B (2002) Different molecular events account for butyrate-induced apoptosis in two human colon cancer cell lines. J Nutr 132, 1812-1818.

Campbell JM, Fahey GC Jr \& Wolf BW (1997) Selected indigestible oligosaccharides affect large bowel mass, cecal and fecal short-chain fatty acids, pH and microflora in rats. $J$ Nutr 127, 130-136.

Cummings JH \& MacFarlane GT (2002) Gastrointestinal effects of prebiotics. Br J Nutr 87, Suppl. 2, S145-S151.

Djouzi Z \& Andrieux C (1997) Compared effects of three oligosaccharides on metabolism of intestinal microflora in rats inoculated with a human faecal flora. Br J Nutr 78, 313-324.

Djouzi Z, Andrieux C, Degivry MC, Bouley C \& Szylit O (1997) The association of yogurt starters with Lactobacillus casei DN 114.001 in fermented milk alters the composition and metabolism of intestinal microflora in germ-free rats and in human flora-associated rats. $J$ Nutr 127, 2260-2266.

Felton JS, Knize MG, Salmon CP, Malfatti MA \& Kulp KS (2002) Human exposure to heterocyclic amine food mutagens/carcinogens: relevance to breast cancer. Environ Mol Mutagen 39, 112-118.

Gérard P, Béguet F, Lepercq P, Rigottier-Gois L, Rochet V, Andrieux C \& Juste C (2004) Gnotobiotic rats, harboring human intestinal microbiota as a model for studying cholesterol-to-coprostanol conversion. FEMS Microbiol Ecol 47, 337-343.

Godon JJ, Zumstein E, Dabert P, Habouzit F \& Moletta R (1997) Molecular microbial diversity of an anaerobic digestor as determined by small-subunit rDNA sequence analysis. Appl Environ Microbiol 63, 2802-2813.

Guérin-Danan C, Chabanet C, Pedone C, Popot F, Vaissade P, Bouley C, Szylit O \& Andrieux C (1998) Milk fermented with yogurt cultures and Lactobacillus casei compared with yogurt and gelled milk: influence on intestinal microflora in healthy infants. Am J Clin Nutr 67, 111-117. 
Hughes R \& Rowland IR (2001) Stimulation of apoptosis by two prebiotic chicory fructans in the rat colon. Carcinogenesis 22, 43-47.

Humblot C, Lhoste EF, Knasmuller S, Gloux K, Bruneau A, Bensaada M, Durao J, Rabot S, Andrieux C \& Kassie F (2004) Protective effect of Brussels sprouts, prebiotics and fermented milk towards IQ-induced genotoxicity in the human flora-associated F344 rat: role of xenobiotic metabolizing enzymes and intestinal microflora. J Chromatogr B Analyt Technol Biomed Life Sci 802, 231-237.

Imaoka A, Setoyama H, Takagi A, Matsumoto S \& Umesaki Y (2004) Improvement of human faecal flora-associated mouse model for evaluation of the functional foods. J Appl Microbiol 96, 656-663.

Johnson IT (2002) Glucosinolates: bioavailability and importance to health. Int $J$ Vitam Nutr Res 72, 26-31.

Kassie F, Lhoste EF, Bruneau A, Zsivkovits M, Ferk F, Uhl M, Zidek T \& Knasmuller S (2004) Effect of intestinal microfloras from vegetarians and meat eaters on the genotoxicity of 2-amino-3-methylimidazo[4,5f]quinoline, a carcinogenic heterocyclic amine. $J$ Chromatogr $B$ Analyt Technol Biomed Life Sci 802, 211-215.

Kassie F, Rabot S, Kundi M, Chabicovsky M, Qin HM \& Knasmüller S (2001) Intestinal microflora plays a crucial role in the genotoxicity of the cooked food mutagen 2-amino-3-methylimidazo[4,5-f]quinoline. Carcinogenesis 22, 1721-1725.

Kassie F, Rabot S, Uhl M, Huber W, Qin HM, Helma C, Schulte-Hermann R \& Knasmüller S (2002) Chemoprotective effects of garden cress (Lepidium sativum) and its constituents towards 2-amino-3-methyl-imidazo[4,5-f]quinoline (IQ)-induced genotoxic effects and colonic preneoplastic lesions. Carcinogenesis 23, 1155-1161.

Kassie F, Uhl M, Rabot S, Grasl-Kraupp B, Verkerk R, Kundi M, Chabicovsky M, Schulte-Hermann R \& Knasmuller S (2003) Chemoprevention of 2-amino-3-methylimidazo[4,5-f]quinoline (IQ)-induced colonic and hepatic preneoplastic lesions in the F344 rat by cruciferous vegetables administered simultaneously with the carcinogen. Carcinogenesis 24, 255-261.

Kimura K, McCartney AL, McConnell MA \& Tannock GW (1997) Analysis of fecal populations of bifidobacteria and lactobacilli and investigation of the immunological responses of their human hosts to the predominant strains. Appl Environ Microbiol 63, 3394-3398.

Kleessen B, Hartmann L \& Blaut M (2001) Oligofructose and long-chain inulin: influence on the gut microbial ecology of rats associated with a human faecal flora. Br J Nutr 86, 291-300.

Knasmüller S, Steinkellner H, Hirschl AM, Rabot S, Nobis EC \& Kassie F (2001) Impact of bacteria in dairy products and of the intestinal microflora on the genotoxic and carcinogenic effects of heterocyclic aromatic amines. Mutat Res 480-481, 129-138.

Krul C, Humblot C, Philippe C, Vermeulen M, van Nuenen M, Havenaar R \& Rabot S (2002) Metabolism of sinigrin (2-propenyl glucosinolate) by the human colonic microflora in a dynamic in vitro large-intestinal model. Carcinogenesis 23, 1009-1016.

Lankaputhra WE \& Shah NP (1998) Antimutagenic properties of probiotic bacteria and of organic acids. Mutat Res 397, 169-182.

Lhoste EF, Ouriet V, Bruel S, Flinois JP, Brézillon C, Magdalou J, Chèze C \& Nugon-Baudon L (2003) The human colonic microflora influences the alterations of xenobiotic-metabolizing enzymes by catechins in male F344 rats. Food Chem Toxicol 41, 695-702.

Muyzer G, de Waal EC \& Uitterlinden AG (1993) Profiling of complex microbial populations by denaturing gradient gel electrophoresis analysis of polymerase chain reaction-amplified genes coding for $16 \mathrm{~S}$ rRNA. Appl Environ Microbiol 59, 695-700.

Muyzer G \& Smalla K (1998) Application of denaturing gradient gel electrophoresis (DGGE) and temperature gradient gel electrophoresis (TGGE) in microbial ecology. Antonie Van Leeuwenhoek 73, 127-141.

Orrhage K, Sillerstrom E, Gustafsson JA, Nord CE \& Rafter J (1994) Binding of mutagenic heterocyclic amines by intestinal and lactic acid bacteria. Mutat Res 311, 239-248.

Rabot S, Szylit O, Nugon-Baudon L, Meslin JC, Vaissade P, Popot F \& Viso M (2000) Variations in digestive physiology of rats after short duration flights aboard the US space shuttle. Dig Dis Sci 45, $1687-1695$.

Reddy BS \& Rivenson A (1993) Inhibitory effect of Bifidobacterium longum on colon, mammary, and liver carcinogenesis induced by 2-amino-3-methylimidazo[4,5-f]quinoline, a food mutagen. Cancer Res 53, 3914-3918.

Roberfroid MB (2001) Prebiotics: preferential substrates for specific germs? Am J Clin Nutr 73, 406S-409S.

Roland N, Rabot S \& Nugon-Baudon L (1996) Modulation of the biological effects of glucosinolates by inulin and oat fibre in gnotobiotic rats inoculated with a human whole faecal flora. Food Chem Toxicol 34, 671-677.

Rouzaud G, Rabot S, Ratcliffe B \& Duncan AJ (2003) Influence of plant and bacterial myrosinase activity on the metabolic fate of glucosinolates in gnotobiotic rats. Br J Nutr 90, 395-404.

Rowland IR, Rumney CJ, Coutts JT \& Lievense LC (1998) Effect of Bifidobacterium longum and inulin on gut bacterial metabolism and carcinogen-induced aberrant crypt foci in rats. Carcinogenesis 19, 281-285.

Satokari RM, Vaughan EE, Akkermans AD, Saarela M \& de Vos WM (2001) Bifidobacterial diversity in human feces detected by genusspecific PCR and denaturing gradient gel electrophoresis. Appl Environ Microbiol 67, 504-513.

Schwab CE, Huber WW, Parzefall W, Hietsch G, Kassie F, SchulteHermann R \& Knasmüller S (2000) Search for compounds that inhibit the genotoxic and carcinogenic effects of heterocyclic aromatic amines. Crit Rev Toxicol 30, 1-69.

Seksik P, Rigottier-Gois L, Gramet G, Sutren M, Pochart P, Marteau P, Jian R \& Doré J (2003) Alterations of the dominant faecal bacterial groups in patients with Crohn's disease of the colon. Gut 52, 237-242.

Suau A, Bonnet R, Sutren M, Godon JJ, Gibson GR, Collins MD \& Doré J (1999) Direct analysis of genes encoding 16S rRNA from complex communities reveals many novel molecular species within the human gut. Appl Environ Microbiol 65, 4799-4807.

Tannock GW (2001) Molecular assessment of intestinal microflora. Am J Clin Nutr 73, 410S-414S.

Tannock GW, Munro K, Harmsen HJ, Welling GW, Smart J \& Gopal PK (2000) Analysis of the fecal microflora of human subjects consuming a probiotic product containing Lactobacillus rhamnosus DR20. Appl Environ Microbiol 66, 2578-2588.

Tavan E, Cayuela C, Antoine JM, Trugnan G, Chaugier C \& Cassand P (2002) Effects of dairy products on heterocyclic aromatic amineinduced rat colon carcinogenesis. Carcinogenesis 23, 477-483.

Vanhoutte T, Huys G, De Brandt E \& Swings J (2004) Temporal stability analysis of the microbiota in human feces by denaturing gradient gel electrophoresis using universal and group-specific 16S rRNA gene primers. FEMS Microbiol Ecol 48, 437-446.

Vaughan EE, Schut F, Heilig HG, Zoetendal EG, de Vos WM \& Akkermans AD (2000) A molecular view of the intestinal ecosystem. Curr Issues Intest Microbiol 1, 1-12.

Walter J, Hertel C, Tannock GW, Lis CM, Munro K \& Hammes WP (2001) Detection of Lactobacillus, Pediococcus, Leuconostoc, and Weissella species in human feces by using group-specific PCR primers and denaturing gradient gel electrophoresis. Appl Environ Microbiol 67, 2578-2585.

Zhang XB \& Ohta Y (1993) Antimutagenicity of cell fractions of microorganisms on potent mutagenic pyrolysates. Mutat Res 298, 247-253.

Zoetendal EG, Akkermans ADL, Akkermans-van Vliet WM, Visser JAGMd \& Vos WMd (2001) The host genotype affects the bacterial community in the human gastrointestinal tract. Microb Ecol Health Dis 13, 129-134

Zoetendal EG, Akkermans AD \& De Vos WM (1998) Temperature gradient gel electrophoresis analysis of 16S rRNA from human fecal samples reveals stable and host-specific communities of active bacteria. Appl Environ Microbiol 64, 3854-3859.

Zsivkovits M, Fekadu K, Sontag G, Nabinger U, Huber WW, Kundi M, Chakraborty A, Foissy H \& Knasmuller S (2003) Prevention of heterocyclic amine-induced DNA damage in colon and liver of rats by different lactobacillus strains. Carcinogenesis 24, 1913-1918. 